\title{
LAS REGIONES INDÍGENAS Y EL MODELO DE LAS UNIVERSIDADES TECNOLÓGICAS EN EL ESTADO DE HIDALGO
}

\author{
Marco Antonio RamíreZ-MartíneZ* \\ Recebido: 28 mar. $2012 \quad$ Aprovado: 26 abr. 2012
}

* Universidad Autónoma del Estado de Hidalgo, México. Estado de Hidalgo, México. Contato com autor: ramirezma23@hotmail.com

Resumen :Este articulo es un acercamiento al desarrollo y funcionamiento del sistema de educación tecnológica en México en los últimos años, denota un panorama general de la evolución de las Universidades Tecnológicas en el país, considerando las políticas y reformas emprendidas por el gobierno federal; situación que ha llevado a este subsistema operar en forma coordinada con los gobiernos de los estados; en cuanto a su factibilidad, creación, presupuesto e instalación de las Universidades Tecnológicas en las distintas regiones. A partir de esto se aborda un análisis de la eficiencia del modelo y sus resultados en la inserción laboral y su impacto social en las regiones donde fueron instaladas estas universidades, donde una de las características principales de estos lugares, fue la marginación social presente en varios ámbitos. Surgen las Universidades Tecnológicas y su vinculación con el sector productivo incipiente en algunos casos, como una única alternativa educacional para los jóvenes egresados del nivel medio superior, que fincan sus anhelos y futuro social en la formación tecnológica; el articulo pretende resaltar el funcionamiento del modelo, reflexionando sobre el papel de todos los involucrados.

Palabras clave: educación superior tecnológica, México, institutos tecnológicos, universidades tecnológicas, universidades politécnicas, regiones indígenas, inserción laboral.

\section{THE INDIAN REGIONS AND THE MODEL OF TECHNOLOGICAL UNIVERSITIES IN THE STATE OF HIDALGO}

Abstract: This article is an approach to the development and operation of technological education in Mexico in recent years. It presents an overview of the evolution of technological universities in the country, considering the policies and reforms undertaken by the federal government, a situation that has led this subsystem to operate in coordination with the state governments in terms of feasibility, creation, budget and installation of technological universities in different regions. This analysis addresses the efficiency of the model and its results in the employment and social impact in the regions where the universities were installed, where one of the main characteristics of these places, social marginalization was present in several areas. The installation of Technological Universities and their linkage with the emerging productive sector in some cases become the sole educational alternative for young high school graduates, that place their hopes and social future in technological training. The article aims to highlight the operation of this educational model, reflecting on the role of all involved .

Keywords : technology higher education in Mexico, technological institutes, technical universities, universities, polytechnics, indigenous areas, employment .

\section{AS REGIÕES INDÍGENAS E O MODELO DAS \\ UNIVERSIDADES TECNOLÓGICAS NO ESTADO DE HIDALGO}

Resumo: Este artigo é uma abordagem para o desenvolvimento e operação de educação tecnológica no México nos últimos anos, denota uma visão geral da evolução das universidades tecnológicas no país, considerando-se as políticas e reformas empreendidas pelo governo federal, uma situação que tem levado este subsistema a operar em coordenação com os governos estaduais, em termos de viabilidade, criação, orçamento e instalação de universidades tecnológicas em diferentes regiões. A partir desta análise aborda a eficiência do modelo e seus resultados no emprego e impacto social nas regiões onde foram instaladas estas universidades, onde uma das principais 
características desses lugares, a marginalização social esteve presente em várias áreas. Surgem as Universidades Tecnológicas e sua vinculação com o setor produtivo emergente, em alguns casos, como uma única alternativa educacional para jovens graduados do ensino médio, que depositam suas esperanças e futuro social na formação tecnológica. O artigo tem como objetivo destacar

o funcionamento deste modelo educacional, refletindo sobre o papel de todos os envolvidos .

Palavras-chave : tecnologia de ensino superior, no México, institutos tecnológicos, universidades técnicas, universidades politécnicas, áreas indígenas, emprego.

\section{INTRODUCCIÓN}

"La educación tecnológica en México está ligada a tres elementos básicos" (ANUIES, 2000).

a) El desarrollo económico.

b) La necesidad de personal capacitado en la industria regional, la infraestructura existente, los servicios públicos que propicien el desarrollo.

c) La Secretaría de Educación Pública (SEP) y los subsistemas de instituciones de educación y enseñanza, a partir de la descentralización federalizada.

En septiembre de 2011, las Universidades Tecnológicas cumplen 20 años en México y también en nuestro estado; entre las características más importantes cabe destacar que desde su creación, estas se han visto cumplidas:

a) Diversificar la estructura educativa para posibilitar una integración al mercado laboral de sus estudiantes y establecer una vinculación con los sectores productivos de las localidades donde se encuentran.

b) Crear opciones académicas de nivel superior, diferentes a las tradicionales.

c) Descentralizar los servicios educativos a nivel superior.

d) Incluir en las políticas educativas estrategias orientadas a subsanar los problemas escolares, académicos y económicos.

Para el año 2011 existen 66 Universidades Tecnológicas en todo el país y cinco se encuentran en el estado de Hidalgo, este subsistema educativo es origen de un intenso proceso dinámico de industrialización y modernización como resultado del Tratado de Libre Comercio de 1994, con un crecimiento y mejoramiento continuo de la economía en los centros urbanos (CGTU y 38 Universidades Tecnológicas, 2000).

Aunado a este proceso, a través del tiempo se ha hecho necesario un incremento de centros educativos tecnológicos y de formación profesional de nuevas 
generaciones de jóvenes para compensar la demanda de técnicos y de personal preparado para trabajar en la organización, manejo, operación y mantenimiento de las factorías industriales y empresas de servicios; principalmente en la industria de la transformación regional y nacional.

Los orígenes de este modelo los podemos encontrar en países desarrollados como Canadá, Estados Unidos y Francia que desde hace años imparten programas de dos años de educación superior tecnológica, cuyo atributo principal es desarrollar las destrezas especificas de una profesión; su creación en México inicia en 1991 con la constitución del Subsistema de Universidades Tecnológicas, cuyo objetivo principal es ofrecer educación superior de dos años, como primer ciclo de educación terciaria según la clasificación de (nivel 5b) UNESCO (2006). La característica fundamental de los planes de estudio es que contemplan un $70 \%$ de formación práctica y un 30\% en la parte teórica.

Considerando esta situación, la SEP en el transcurso de la última década del siglo pasado, analizó, creó y transformó las instituciones de enseñanza tecnológica de acuerdo a esta nueva modalidad. De esta forma se garantizó la educación tecnológica creando el "Sistema Nacional de Institutos Tecnológicos" (SNIT), que incluye a todas las regiones del país. En el marco de una actividad establecida como prioridad, se creó una red de escuelas; universidades públicas y particulares, universidades tecnológicas de reciente creación, institutos tecnológicos y escuelas politécnicas, con un contenido y programas en estudios prácticos y experimentales, concentrados en la educación tecnológica. Bajo este modelo, acertadamente se crearon las Universidades Tecnológicas en el estado de Hidalgo, región central de México (COORDINACIÓN GENERAL DE UNIVERSIDADES TECNOLÓGICAS - CGUT, 2006).

En el estado de Hidalgo, una función social especial en este entorno de bajos recursos y mediano desarrollo empresarial, la tienen las Universidades Tecnológicas "UT", quienes presentan programas académicos de educación superior de calidad, que tienden a responder a las exigencias del sector productivo regional. Este modelo de Universidad promueve y facilita la vinculación empresarial e industrial para certificar los saberes prácticos y solucionar en alguna medida el acceso de sus egresados al mercado de trabajo (CGTU y 38 Universidades Tecnológicas, 2000).

La implementación del modelo de las universidades tecnológicas en el país, inicia en 1991 con la creación de tres centros educativos, en ciudad Netzahualcóyotl en el estado de México, en la ciudad de Aguascalientes en Aguascalientes y en Tula-Tepeji, en el estado de Hidalgo la Universidad Tecnológica de Tula Tepeji; la población de las tres universidades ascendía a 426 estudiantes en 
ese año. Ofreciendo estas instituciones la titulación de técnicos superiores universitarios, que corresponde al nivel 5B, de acuerdo a la clasificación de ciclos educativos de la UNESCO (CGUT, 2004). El plan de estudios consta de seis cuatrimestres, es decir dos años. Durante el desarrollo del plan de estudios, los estudiantes llevan a cabo una estadía en los sectores industrial y de servicios, durante quince semanas que equivale a un cuatrimestre, para completar su formación y otorgarles el título.

En la primera década del siglo veintiuno, el debate sobre el futuro de la educación superior ha estado presente en diversos foros. Se ha hecho evidente revisar en los países en vías de desarrollo la operación de sus sistemas educativos, transformarlos y adecuarlos a las necesidades coligadas con la globalización y las perspectivas de crecimiento de estos países. Las autoridades educativas y las propias Universidades Tecnológicas del Estado de Hidalgo coinciden en señalar la importancia estratégica de la educación como medio fundamental para generar el desarrollo sostenible de la sociedad.

Iniciamos la segunda década del siglo veintiuno dentro de la estructura plena de una sociedad del conocimiento, en la cual la exigencia educativa es mayor no sólo en términos de los niveles de educación formal de la población sino de la educación y formación durante toda la vida (RUIZ-LARRAGUIVEL, 2011).

\section{EL MODELO EDUCATIVO TECNOLÓGICO}

El modelo de las Universidades Tecnológicas es resultado de experiencias de países con mayor crecimiento económico y bienestar social, México se desarrolló este modelo educativo con la finalidad de brindar un servicio a los sectores productivos, articulando la educación con la empresa, permitiendo a los estudiantes cumplir satisfactoriamente sus expectativas de formación académica y desempeño profesional. Consecuentemente en 1991 se crea fundamentado en el modelo educativo tecnológico francés, el Sistema de Universidades Tecnológicas, basado en un modelo pedagógico que, por sus principios reorienta a la calidad, pertinencia, intensidad, continuidad, polivalencia y flexibilidad de los cursos, para dotar a los educandos de un título universitario como técnico superior (CGTU y 38 Universidades Tecnológicas, 2000).

\section{DESARROLLO DEL MODELO EN EL ESTADO DE HIDALGO}

La educación en tecnología es un área de estudio relativamente nueva en el estado de Hidalgo, México; con aproximadamente 25 años. La educación en 
tecnología sitúa al estudiante en el ámbito laboral con experiencias y métodos que aumentan la comprensión de los procesos tecnológicos y como estos afectan su vida frente a los cambios educativos que están sucediendo; el modelo de la educación tecnológica se exterioriza como una alternativa a la educación en los municipios y regiones donde se encuentran estas universidades. En el nuevo paradigma de educación en tecnología existen cambios de valores y afirmaciones; la forma de ver la preparación técnica universitaria, sus métodos y los logros que se pueden obtener, difieren de los saberes industriales y manufacturas tradicionales. El contenido del currículo está diseñado con la finalidad de servir a las demandas de los estudiantes en cuanto al desarrollo de una cultura e interactividad tecnológica educativa.

Figura 1 - Planes de Estudio. Universidades Tecnológicas

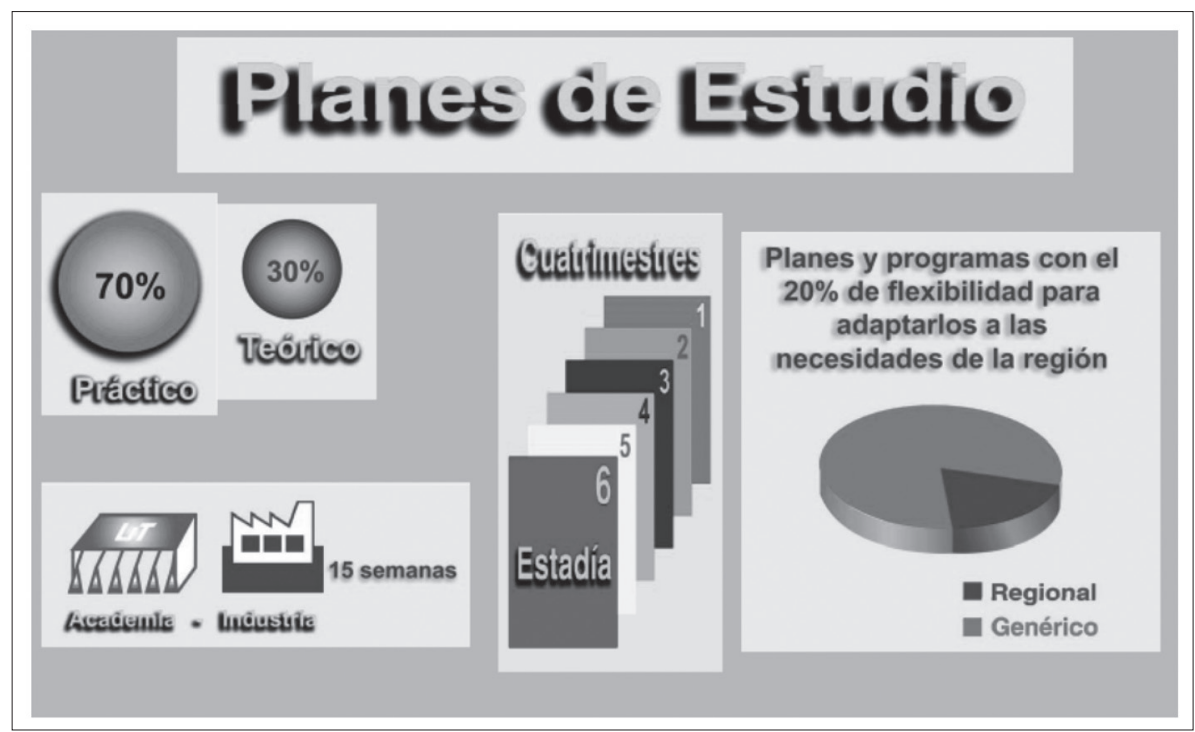

Fuente: Coordinación General de Universidades Tecnológicas (CGUT)

En el estado de Hidalgo se han creado cinco Universidades Tecnológicas: Universidad Tecnológica Tula-Tepeji, Universidad Tecnológica de Tulancingo, Universidad Tecnológica del Valle del Mezquital, Universidad Tecnológica de la Huasteca Hidalguense, Universidad Tecnológica de la Sierra Hidalguense.

Con una "matricula total en 2009 de 5,028" (SEP, 2010) alumnos en las diferentes áreas y carreras que se ofrecen, siendo de las más importantes en el país, tanto por su matrícula como por sus resultados. 
Cuadro 1 - Matricula Universidades Tecnológicas 2008-9

\begin{tabular}{|c|c|c|c|c|c|}
\hline \multicolumn{6}{|c|}{ POBLACIÓN ESCOLAR TOTAL , 2008-2009 } \\
\hline \multicolumn{6}{|c|}{ (INGRESO, REINGRESO, EGRESADOS Y TITULADOS) } \\
\hline \multirow{2}{*}{ ENTIDAD FEDERATIVA } & PRIMER & \multirow{2}{*}{ REINGRESO } & MATRICULA & EGRESADOS & \multirow{2}{*}{$\begin{array}{c}\text { TITULADOS } \\
2007-2008 \\
\end{array}$} \\
\hline & INGRESO & & TOTAL & $2007-2008$ & \\
\hline AGUASCALIENTES & 1,700 & 1,056 & 2,756 & 724 & 651 \\
\hline BAJA CALIFORNIA & 867 & 567 & 1,434 & 428 & 413 \\
\hline \multicolumn{6}{|l|}{ BAJA CALIFORNIA SUR } \\
\hline CAMPECHE & 909 & 667 & 1,576 & 552 & 103 \\
\hline COAHUILA & 2,779 & 2,268 & 5,047 & 1,365 & 1,196 \\
\hline COLIMA & 139 & 254 & 393 & 32 & 34 \\
\hline CHIAPAS & 995 & 580 & 1,575 & 549 & 519 \\
\hline CHIHUAHUA & 1,593 & 1,929 & 3,522 & 445 & 83 \\
\hline DISTRITO FEDERAL & 505 & 480 & 985 & 337 & 203 \\
\hline \multicolumn{6}{|l|}{ DURANGO } \\
\hline GUANAJUATO & 3,166 & 2,680 & 5,846 & 1,724 & 1,588 \\
\hline GUERRERO & 1,307 & 848 & 2,155 & 682 & 396 \\
\hline HIDALGO & 2,667 & 2,361 & 5,028 & 1,473 & 1,471 \\
\hline JALISCO & 1,745 & 3,287 & 5,032 & 1,490 & 1,147 \\
\hline MEXICO & 5,222 & 5,483 & 10,705 & 2,352 & 1,979 \\
\hline MCHOACANN" & 334 & 190 & 524 & 182 & 103 \\
\hline MOR日 OS & 698 & 954 & 1,652 & 362 & 272 \\
\hline NAYARI & 1,822 & 1,180 & 3,002 & 856 & 596 \\
\hline NUEVO LEÓN & 1,191 & 1,506 & 2,697 & 709 & 624 \\
\hline \multicolumn{6}{|l|}{ OAXACA $^{*}$} \\
\hline PUEBA & 4,705 & 3,958 & 8,663 & 2,333 & 2,047 \\
\hline QUERETARO & 2,046 & 2,326 & 4,372 & 1,475 & 1,347 \\
\hline QUINTANA ROO & 1,431 & 1,020 & 2,451 & 143 & 78 \\
\hline SAN LUIS POTOSI & 746 & 591 & 1,337 & 393 & 32 \\
\hline SINALOA & 378 & 472 & 850 & 261 & 14 \\
\hline SONORA & 2,551 & 2,190 & 4,741 & 1,027 & 976 \\
\hline TABASCO & 1253 & 1,491 & 2744 & 1,024 & 190 \\
\hline TAMAULIPAS & 2,021 & 1,959 & 3,980 & 1,178 & 903 \\
\hline TLAXCALA & 565 & 327 & 892 & 229 & 100 \\
\hline VERACRUZ & 1,599 & 1,056 & 2,655 & 580 & 69 \\
\hline YUCATAN & 1,773 & 1,883 & 3,656 & 913 & 886 \\
\hline ZACATECAS & 628 & 632 & 1,260 & 380 & 349 \\
\hline & & & & & \\
\hline TOTAL NACIONAL & 47,335 & 44,195 & 91,530 & 24,198 & 18,369 \\
\hline
\end{tabular}

Fuente: SEP. Anuario estadístico. México: ANUIES, 2010.

Si bien el sistema en el estado de Hidalgo ha sido eficaz para atraer a los jóvenes a sus aulas y para facilitar el tránsito de la escuela al trabajo, se deben hacer esfuerzos institucionales y políticos para ofrecer la posibilidad real de continuar sus estudios superiores, de ser posibles en las propias Universidades Tecnológicas UT's modalidad que se ofrece desde el año 2010, y así cerrar un ciclo ó modelo educativo, en las mismas regiones donde están ubicadas.

El reto de este modelo, fue definir bajo el concepto tecnológico, cuáles tecnologías, cómo y por qué se estudiarán y sobre todo la aplicación que puedan tener en el Estado de Hidalgo, en el caso de la fundación de sus UT's 
en los años noventas principalmente en las regiones indígenas. Este modelo supone y genera una forma conjunta administrativa del gobierno en materia de educación e industria, de realizar inversiones que propicien empleos coherentes a los resultados de la Educación tecnológica. La sociedad demanda de las instituciones educativas una oferta de cultura científica y tecnológica para los nuevos ciudadanos con la confianza de que eso es algo importante, pero, una vez hecha esa demanda, no pone condiciones a su cumplimiento, sino que, en cierto modo, entrega un documento en blanco a quienes diseñan los currículos tecnológicos, considerando que su papel como expertos garantizará que sabrán articular de la mejor manera las enseñanzas de la ciencias y la tecnología. Con esta solicitud al sistema educativo científico y tecnológico, la sociedad se relaciona con ella de un modo no muy diferente al que caracterizó a los modelos lineales de desarrollo de la ciencia y la tecnología promovidos por la SEP en los años 70, y que tan anacrónicos se han demostrado con el paso del tiempo.

Por supuesto el modelo de educación tecnológica en el estado de Hidalgo, es decir el funcionamiento de las cinco Universidades Tecnológicas no es ajeno a la problemática, a los nuevos planteamientos o reformas educativas nacionales que influyen en su funcionamiento y su relación con los demás niveles educativos y con posibles cambios en la industria de la región.

A este respecto, con la intención de clasificar a las UT's de Hidalgo por importancia en la integración de sus egresados a la planta laboral, solo las universidades de Tula-Tepeji, Universidad Tecnológica del Valle del Mezquital y en menor medidas las otras tres, se han acercado a los objetivos finales para las que fueron creadas (RAMSEY, 2000).

En el caso particular de Hidalgo, las reformas educativas presentan algunas situaciones que inciden en el quehacer diario de las UT's. Una de ellas es la separación en subsistemas educativos y el grado de centralización que interviene en la coordinación de esfuerzos de mejoramiento; por el origen de los recursos financieros que reciben estas. En el caso de las UT's de Hidalgo algunas problemáticas se derivan particularmente de la naturaleza organizacional y cultural de las instituciones educativas y su contexto. Algunos rasgos estructurales en este proceso se están contextualizando regionalmente.

Por un lado se espera que favorezcan la conservación de la cultura y tradiciones de la localidad pero, por el otro, se espera que apoyen el cambio social y orienten su aprendizaje hacia una plataforma productiva global pero considerando las características propias del contexto local. En el desarrollo de un modelo y su extensión hacia la sociedad o mejor dicho el impacto social que 
Figura 2 - El modelo y sus objetivos

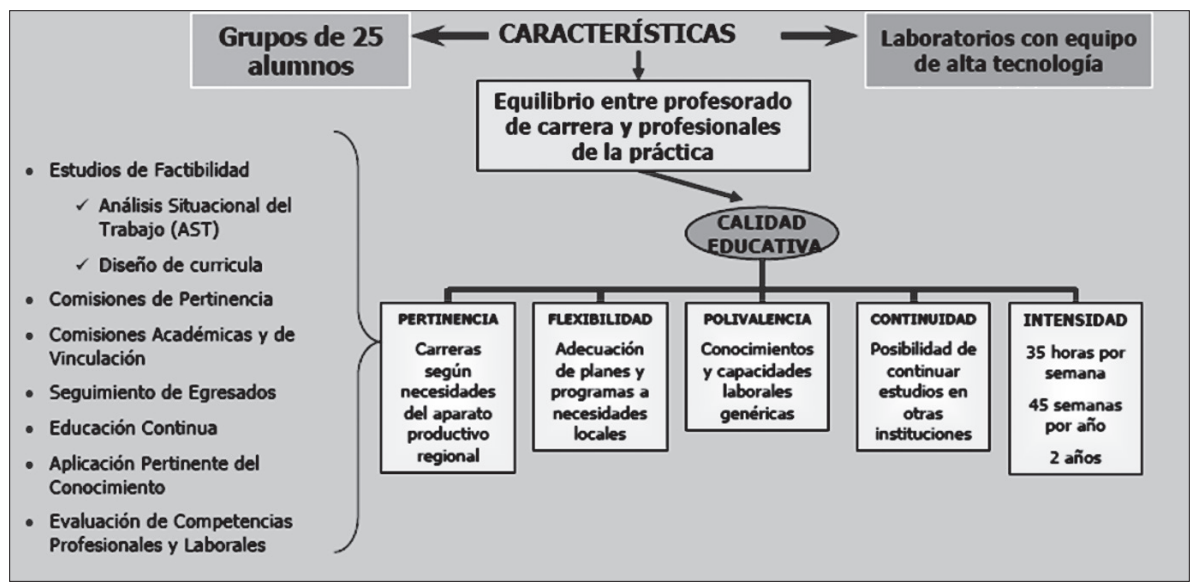

Fuente: Coordinación General de Universidades Tecnológicas (CGUT)

pueda tener, donde lo más importante son los resultados locales, es decir, la valoración del modelo por la sociedad; el conocimiento y el compromiso en los jóvenes, son entidades que no se pueden asignar como tales, sólo desarrollar. Uno de los retos de este modelo de educación tecnológica es crear las condiciones necesarias para movilizar a los actores institucionales, especialmente a los profesores para que constituyan como un recurso a favor de éstas condiciones, porque la realidad es que sin una integración real docente con la misión y visión de cada una de nuestras universidades tecnológicas, las intenciones y objetivos del modelo se ven reducidas a reproducir prácticas de modelos tradicionales.

El éxito de este modelo está relacionado con el hecho de que los actores le encuentren utilidad y significado social como agente de cambio en la localidad o región. Que quienes participan construyan un concepto viable acerca de la transformación del entorno; esta perspectiva es muy importante porque les permite reflexionar y tener una postura en relación a éste modelo, ya sea la condición de postura de instituirlo cabalmente, objetarlo y utilizarlo hacia sus interés (LORENZA VILLA LEVER, 2002).

Con respecto a el compromiso social podemos decir que este modelo permite la participación de diferentes actores de la sociedad, autoridades de gobierno locales y estatales, autoridades educativas y el sector productivo, están representados en la toma de decisiones y asignación de recursos a los distintos proyectos; este aspecto es coincidente con el modelo de las Universidades Tecnológicas 
de Francia, donde la participación empresarial y de otras universidades está involucrada en el funcionamiento de estas.

El funcionamiento del Modelo de la Universidades Tecnológicas en el estado de Hidalgo, no ha sido ajeno a el resto de los institutos Tecnológicos de la república mexicana; su expansión local tiene sus bases en el sistema de educación superior tecnológica, después de una reforma educativa, denominada "Reforma de la Educación Superior Tecnológica" misma que se concibe como una política sectorial necesaria en las nuevas condiciones tecnológicas del país y ubicada en el contexto de la modernización económica (RUIZ-LARRAGUIVEL, 2011). Las Universidades Tecnológicas nacen con la consigna de orientarse al conocimiento aplicado y dar respuesta a las necesidades de la industria local y regional, la reforma educativa parece colocar en cuestión este propósito. De alguna manera, la reforma trazó cambios de diversa índole; en la finalidad de la educación, en los medios utilizados, cambios tecnológicos y estructurales, también en la relación con el entorno en su conjunto. Cabe señalar que no se concretaron tal como estaba planeado, pero que sin embargo se han realizado importantes transformaciones a lo largo de veinte años, que propician un mejor funcionamiento del modelo y que además, promete que los actores educativos comprendan la potencialidad de este modelo en beneficio de las regiones indígenas donde están ubicadas.

La innovación implica que verdaderamente se puedan observar y llevar cabo modificaciones desde las posiciones directivas tanto en las prácticas académicas como administrativas. La reforma educativa de los años noventa implicó modificaciones en algunos ámbitos de la educación tecnológica, tales como nuevas ingenierías, licenciaturas y técnicos universitarios acordes al desarrollo tecnológico mundial, encausar el servicio social en beneficio del aprendizaje técnico, estadías y residencias profesionales; mientras que en otros ámbitos se siguieron utilizando las mismas prácticas y formas administrativas. Entonces surge la problemática de cómo consolidar la propuesta de reorganizar el sistema, como se presenta el caso del sistema de educación tecnológica, sus diferentes modalidades, cuyos resultados se deben reflejar cualitativamente en la sociedad.

\section{METODOLOGÍA}

La intención de este ensayo es propiciar la reflexión acerca de la importancia de considerar si el modelo propuesto en un inicio para las Universidades Tecnológicas del estado de Hidalgo ha funcionado como se esperaba; aun cuando haya sido objeto de reformas como parte de un subsistema nacional. Para este 
fin se utilizó una metodología descriptiva, exploratoria, teniendo como punto de partida documentos, datos y eventos relacionados, para conocer cómo es y se manifiesta este modelo educativo en el estado de Hidalgo. Bajo esta misma línea de análisis se propone reflexionar sobre la necesidad de evaluar con anticipación si las instituciones singulares tienen las condiciones necesarias para implantar un modelo tecnológico integral. Describiendo las condiciones actuales del funcionamiento, operación y aceptación social de las universidades tecnológicas que se encuentran en el estado de Hidalgo; que aporten elementos para la reflexión y análisis hacia el futuro de la educación tecnológica.

\section{ANÁLISIS}

En México, los argumentos para crear el sistema de UT's fueron; impulsar la formación vocacional o profesional, diversificar la oferta y ofrecer estudios de nivel superior en regiones con relativa desventaja económica y social, lo que parece responder a la necesidad de incidir con estrategias concretas, hacia los problemas creados por la inequidad social en nuestro país y que se reflejan en los centros educativos.

Por otro lado, de acuerdo con los datos oficiales el costo de la formación del técnico superior universitario ha disminuido con el tiempo, "Entre 1995 y 2000, el promedio por alumno disminuyó, de 59.11 miles de pesos a 26.62 miles de pesos, aunque, se agrega, que en 1999, las primeras universidades ya tenían un costo por alumno menor, que oscilaba entre los 15 mil y los 17 mil pesos" (RAMSEY, 2000). Con base en sus propios cálculos el estudio realizado por Ramsey, Carnoy y Woodburne (2000), afirma que las universidades tecnológicas gastan por estudiante al año casi tanto como otras universidades. No obstante, el Observatorio Ciudadano de la Educación (OCE), muestra que el costo anual por alumno cambia entre universidades, por carrera y por año, dependiendo de la matrícula y del desarrollo de cada institución pero que, actualmente, el gasto federal promedio por estudiante en una UT es superior al de las otras universidades públicas que según el Informe en 2003 de la Secretaría de Educación Pública invertían 15 mil 648.4 (CRESPO, 2009) pesos por alumno, siendo esto un promedio nacional, donde están incluidas las Universidades tecnológicas del Estado de Hidalgo.

No obstante, para la Dirección de Evaluación y Prospectiva el costo mayor de los Institutos Universitarios Tecnológicos es justificable, en la medida en la que tienen una alta tasa de eficiencia terminal superior ${ }^{1} \mathrm{y}$ sus egresados se

1 Véase Cuadro 1 La matriculación de alumnos y egresados titulados 
insertan con facilidad en el mercado de trabajo, al menos en el corto plazo. En otras palabras, lo importante no es cuánto se gasta, sino que el resultado de este modelo educativo sea considerado de calidad satisfactoria.

Algunos de los elementos más importantes que coadyuvan al alza de los costos de la educación impartida en los modelos analizados son la disminución del número de alumnos por profesor, el hecho de que el equipo tecnológico no se comparta con otras instituciones de educación técnica superior cercanas, la política de localización geográfica orientada hacia las ciudades con menor infraestructura, que aumenta los costos en equipo y personal; o la tendencia cada vez más fuerte a que los egresados continúen estudios universitarios.

Estos argumentos hacen suponer que la inversión en educación no se traduce en resultados cualitativamente importantes, sin embargo, la inversión educativa tiene la capacidad de mejorar la calidad de vida y en ese sentido, tiene un trascendente potencial para propiciar la equidad social en las regiones indígenas. Pero mientras no se mejore la eficiencia y la eficacia del sistema de Universidades Tecnológicas, éste no podrá ser un promotor real de la equidad social. Un punto interesante a distinguir es el destino de los egresados; es preciso mencionar que, a pesar del alto nivel de inserción de sus egresados al mercado de trabajo, las Universidades Tecnológicas sólo han logrado captar la atención de algunos sectores de la población local, particularmente hijos de familias de ingresos medios a medios bajos. Este hecho está interpretado como un rasgo conservador de los mexicanos, ya que las familias creen, que las carreras tradicionales, aseguran el bienestar de sus hijos (ANUIES, 2000).

En México los trabajadores con más altos grados de escolaridad han visto, en los últimos años, aumentar sus ingresos, así como han disminuido los de aquellos, con una escolaridad menor. El hecho es que no es equívoco afirmar que durante mucho tiempo se ha cumplido, en nuestro país, con la premisa de "a mayor nivel educativo, mejores condiciones laborales y de ingreso", particularmente a partir de que el Estado impulsa el desarrollo industrial y hasta que el modelo de desarrollo deje de responder a las necesidades de la población. El acceso al mercado laboral, en condiciones acordes con el nivel de calificación, era entonces posible porque hasta los años ochentas eran muy pocos los jóvenes que tenían oportunidad de seguir estudios universitarios, mientras que la economía crecía con rapidez y ocupaba recursos humanos formados.

Es por ello que se puede decir que los egresados universitarios tenían, en los años ochenta, prácticamente asegurado el empleo, por lo que entre 
ciertos grupos de la sociedad mexicana perdura la idea de que el futuro de los jóvenes es más seguro si se hacen los estudios en las universidades tradicionales que si se elige una opción de educación superior corta, nueva, poco conocida y sin un posicionamiento muy claro en el mercado de trabajo, como las UT's. Actualmente la educación superior técnica en el Estado de Hidalgo está incorporando a grupos sociales antes excluidos del nivel superior, permitiéndoles acceder a posiciones laborales que, a su vez, les generan beneficios económicos antes difícilmente alcanzables para ellos. Además hay que reconocer la capacidad de los jóvenes para entender cómo funciona realmente el mercado laboral y cuáles son sus reglas. A medida que los Técnicos Superiores Universitarios comprendan los laberintos del mundo de trabajo, ellos mismos sabrán qué estrategias usar para sortear las dificultades de la vida productiva; una conclusión anticipada del funcionamiento del modelo en las regiones indígenas en el estado de Hidalgo, la observamos en la matricula de inscripción a determinadas carreras, que van orientando la pertinencia y aceptación social de las UT's en los procesos de inserción laboral regionales. Como se muestra en el cuadro 2.

No podemos dejar a un lado la comparación con el modelo educativo de origen Francés; en el contexto del Plan Universidad 2000, el gobierno de Francia dio un lugar muy importante a los Institutos Universitarios Tecnológicos, primero, porque consideró que aumentando la matrícula de alumnos podría reducirse el número de estudiantes provenientes de los bachilleratos tecnológicos que se inscriben a la universidad y fracasan. Segundo, porque había interés en abrir nuevas modalidades de formación para técnicos superiores, como resultado del análisis de la evolución del mercado de empleo y de las necesidades de adaptación, es decir, a los sectores secundario y terciario regionales y nacionales.

Como se dijo al principio de este ensayo, la desigualdad socioeconómica es uno de los grandes problemas del Estado de Hidalgo; una de sus más graves manifestaciones es la deserción escolar que, a su vez, es uno de los principales problemas a resolver en la Universidades Tecnológicas. La deserción está ligada a los costos que implica asistir a la universidad, tales como el pago de colegiaturas, libros, transporte, etcétera. Costos directos e indirectos; a este respecto las recomendaciones que hacen algunos analistas con relación a la puesta en marcha de un sistema de becas, así como a la apertura de opciones educativas para los jóvenes que trabajan, son muy importantes para auspiciar la equidad educativa (COORDINACIÓN GENERAL DE UNIVERSIDADES TECNOLÓGICAS , 2006). 
LAS REGIONES INDÍGENAS Y EL MODELO DE LAS UNIVERSIDADES TECNOLÓGICAS EN EL ESTADO DE HIDALGO

Cuadro 2 - Matricula Universidades Tecnológicas por aéreas de estudio. 2008-9

\begin{tabular}{|c|c|c|c|c|c|c|c|c|c|c|c|c|c|c|}
\hline \multicolumn{8}{|c|}{ MA TRÍCULA DEL RÉGIMEN PÚBLICO POR ÁREAS DE ESTUDIO, 2008-2009 } & & & & & & & \\
\hline \multirow[t]{2}{*}{ ENIDAD FEDERATIVA } & \multicolumn{2}{|c|}{$\begin{array}{c}\text { CIENCIAS } \\
\text { AGROPECUARIAS }\end{array}$} & \multicolumn{2}{|c|}{$\begin{array}{l}\text { CIENCIA S DE LA } \\
\text { SALUD }\end{array}$} & \multicolumn{2}{|c|}{$\begin{array}{l}\text { CIENCIAS } \\
\text { NATURALESY } \\
\text { EXACTAS }\end{array}$} & \multicolumn{2}{|c|}{$\begin{array}{c}\text { CIENCIAS } \\
\text { SOCIALESY } \\
\text { ADMINISTRATIVAS }\end{array}$} & \multicolumn{2}{|c|}{$\begin{array}{l}\text { EDUCACIÓN Y } \\
\text { HUMANIDADES }\end{array}$} & \multicolumn{2}{|c|}{$\begin{array}{l}\text { INGENERAAY } \\
\text { TECNOLOGIA }\end{array}$} & \multicolumn{2}{|c|}{ TOTAL } \\
\hline & ABS. & $\%$ & ABS. & $\%$ & ABS. & $\%$ & ABS. & $\%$ & ABS. & $\%$ & ABS. & $\%$ & ABS. & $\%$ \\
\hline AGUASCALENIES & & & 241 & 8.8 & & & 1,264 & 46.4 & 31 & 1.1 & 1,190 & 43.7 & 2,726 & 3.1 \\
\hline BAJA CALFOPNA & & & & & & & 547 & 39.5 & & & 838 & 60.5 & 1,385 & 1.6 \\
\hline \multicolumn{15}{|l|}{ BAJA CALFOPNA SUR } \\
\hline CAMPECEE & 9 & 0.7 & 133 & 10.5 & & & 431 & 34.1 & & & 691 & 54.7 & 1,264 & 1.4 \\
\hline COAHUILA & & & & & & & 1,406 & 280 & & & 3,610 & 720 & 5,016 & 5.7 \\
\hline COLLIMA & & & & & & & 242 & 61.6 & & & 151 & 38.4 & 393 & 0.4 \\
\hline CHAPAS & 246 & 15.6 & & & & & 585 & 37.1 & & & 744 & 47.2 & 1,575 & 1.8 \\
\hline CHHUAHUA & 70 & 20 & 71 & 20 & & & 556 & 15.8 & 182 & 5.2 & 2643 & 75.0 & 3,522 & 4.0 \\
\hline DISTRTO FERAL & & & 27 & 4.1 & & & 550 & 831 & 85 & 128 & & & 662 & 0.8 \\
\hline \multicolumn{15}{|l|}{ DURANGO ${ }^{ \pm}$} \\
\hline GUANAJUATO & 74 & 1.3 & & & & & 2,628 & 46.3 & & & 2,969 & 524 & 5,671 & 6.5 \\
\hline GLEPPERO & & & & & & & 685 & 328 & & & 1,405 & 67.2 & 2,090 & 2.4 \\
\hline HDALGO & 44 & 0.9 & 111 & 22 & & & 2,137 & 425 & & & 2736 & 54.4 & 5,028 & 5.8 \\
\hline JALSOO & & & 709 & 17.5 & & & 943 & 233 & & & 2,401 & 59.2 & 4,053 & 4.6 \\
\hline MÉxICD & & & 996 & 9.6 & & & 3,344 & 322 & 282 & 27 & 5,750 & 55.4 & 10,372 & 11.9 \\
\hline MIOHOACÁN" & & & & & & & & & & & 515 & 100.0 & 515 & 0.6 \\
\hline MORHOS & 108 & 6.5 & & & & & 980 & 59.3 & 59 & 3.6 & 505 & 30.6 & 1,652 & 1.9 \\
\hline NAYART & 161 & 5.4 & & & & & 2,182 & 727 & & & 659 & 220 & 3,002 & 3.4 \\
\hline NUEVOLEÓN & & & 248 & 9.6 & & & 523 & 20.2 & 3 & & 1,812 & 70.1 & 2,586 & 3.0 \\
\hline \multicolumn{15}{|l|}{ OAXACA* } \\
\hline PU⿴囗十 & 242 & 29 & 120 & 1.4 & & & 2,444 & 29.4 & & & 5,517 & 66.3 & 8,323 & 9.5 \\
\hline QLERÉARO & & & & & & & 1,704 & 39.5 & 10 & & 2,604 & 60.3 & 4,318 & 4.9 \\
\hline QUNTANA RDO & & & & & & & 1,901 & 81.3 & 16 & 0.7 & 422 & 18.0 & 2,339 & 2.7 \\
\hline SANLUISPOTOSI & & & & & & & 430 & 322 & 104 & 7.8 & 803 & 60.1 & 1,337 & 1.5 \\
\hline SINALOA & 12 & 1.7 & 607 & 85.9 & & & 37 & 52 & 26 & 37 & 25 & 3.5 & 707 & 0.8 \\
\hline SONORA & & & 200 & 43 & & & 1,538 & 332 & 328 & 7.1 & 2,573 & 55.5 & 4,639 & 5.3 \\
\hline TABASCD & 36 & 1.3 & & & & & 1,469 & 535 & & & 1,239 & 45.2 & 2,744 & 3.1 \\
\hline TAMAUUPAS & & & 239 & 6.0 & & & 1,204 & 30.3 & & & 2,537 & 63.7 & 3,980 & 4.6 \\
\hline TLAXCALA & & & & & & & 367 & 41.1 & & & 525 & 58.9 & 892 & 1.0 \\
\hline VERACRE & 61 & 23 & 337 & 128 & & & 605 & 230 & & & 1,625 & 61.8 & 2,628 & 3.0 \\
\hline YUCATÁN & & & & & & & 1,474 & 50.0 & 245 & 8.3 & 1,227 & 41.6 & 2,946 & 3.4 \\
\hline ZACATECAS & & & 188 & 18.4 & & & 243 & 23.8 & & & 589 & 57.7 & 1,020 & 1.2 \\
\hline TOTAL NA CONAL & $1,063^{\prime}$ & 1.2 & 4,27 & 4.8 & & & $32,419^{\prime}$ & 37.1 & $1,371^{\prime \prime}$ & 1.6 & $48,305^{\prime}$ & 55.3 & 87,385 & 100.0 \\
\hline
\end{tabular}

Fuente: SEP. Anuario estadístico. México: ANUIES, 2010.

Como se describe a continuación, expertos internacionales hicieron una serie de recomendaciones a las Universidades Tecnológicas, que tienen que ver con el diseño de algunas estrategias orientadas a responder a las necesidades del aprendizaje de los estudiantes (LORENZA VILLA LEVER, 2002).

Estas recomendaciones se exponen aquí, clasificadas en tres categorías:
a) institucionales
b) académicas
c) administrativas. 


\section{RECOMENDACIONES AL SUBSISTEMA DE LAS UNIVERSIDADES TECNOLÓGICAS}

\section{a) Institucionales}

1. Dar a conocer el perfil del Técnico Superior Universitario o profesional asociado, y su ubicación en el mercado laboral.

2. Establecer mecanismos para aumentar la cobertura y prevenir la reprobación y la deserción.

3. Instrumentar programas para el otorgamiento de becas.

4. Crear modalidades para estudiantes que trabajan.

5. Resolver los problemas que impiden la continuidad de estudios.

\section{b) Académicas}

6. Disminuir la carga horaria de los estudiantes.

7. Fortalecer la formación de actitudes en los estudiantes.

8. Mantener el "espíritu tecnológico" en la apertura de nuevas carreras.

9. Redefinir el perfil del personal docente.

\section{c) Administrativas}

11. Revisar los procedimientos administrativos y financieros, dando más responsabilidad al nivel local.

12. Actualizar la administración y la dirección interna, en concordancia con el modelo educativo.

\section{CONCLUSIONES}

Los jóvenes que estudian en las UT del Estado de Hidalgo provienen, en general, de un medio socioeconómico en mayor desventaja que aquellos que cursan la educación superior tradicional, principalmente de regiones indígenas. Esto genera un escenario en donde la inversión en la formación de recursos humanos no es suficiente para erradicar los problemas estructurales en términos económicos y sociales. Es indispensable que las políticas educativas integren mecanismos compensatorios con objeto de evitar mayores desigualdades, situación que se ha superado parcialmente en el estado de Hidalgo a través del otorgamiento de diferentes becas a los estudiantes. 
Otra condición importante para que la inversión en educación tecnológica tenga el impacto esperado, en términos económicos y productivos, es la existencia de oportunidades de empleo con una cierta base tecnológica, que pueda aprovechar a un trabajador con el Perfil del Técnico Superior Universitario.

Aunque los sistemas de educación superior corta, tanto de nuestro país como de Francia, nacen de la teoría del capital humano, en el Estado de Hidalgo la desventaja económica subraya las diferencias con sus contrapartes en otros estados del país. A pesar de los dispares niveles de desarrollo, entre nuestro Estado, nuestro país y los países de la OCDE, es claro el beneficio de tener una estructura educativa diversificada que posibilite trayectorias distintas, que sean integralmente incluyentes.

Para asumir un panorama integral, en necesario considerar que la educación superior tecnológica a nivel técnico, ubicada en ciudades medias o en poblaciones relativamente apartadas y de menor desarrollo como las regiones indígenas, enfrenta retos, como los altos costos directos e indirectos que representa estudiar a los alumnos egresados del bachillerato. Por otro lado la dualidad educativa constituida por la obtención de un diploma válido en el mercado de trabajo y la posibilidad de continuar estudios superiores, no ha podido ser alcanzada adecuadamente.

Además, los programas de becas no han sido suficientes para evitar la deserción. Si aun en contextos con condiciones más favorables, los modelos educativos enfrentan problemas que les dificultan ejercer el papel de agentes de cambio en el desarrollo económico regional, mucho menos se puede esperar de las opciones educativas inmersas en contextos socioeconómicos con las características particulares del estado de Hidalgo. De ahí la importancia de que se desarrollen y apliquen políticas económico sociales mucho más coherentes en cada una de las regiones.

El crecimiento estatal de la matrícula de las Universidades Tecnológicas ha mostrado, además del interés del Estado en impulsarlo, que el sistema cubre expectativas de educación y empleo entre algunos jóvenes de las localidades, esencialmente en las regiones indígenas. No obstante, la retención durante todo el proceso educativo no es suficiente y en ello influyen tanto factores económicos, académicos, la alta tasa de reprobación, debido en parte al nivel de formación con el que ingresan los alumnos a las universidades tecnológicas, deserción, dificultades para ser estudiantes de tiempo completo y obstáculos institucionales para continuar estudios superiores; estos son problemas que las UT's deben resolver con políticas educativas precisas, enfocadas a las características de la población de las regiones indígenas, que asiste a cada plantel. 
En síntesis, las políticas educativas estatales deben rescatar el potencial que la educación tecnológica tiene para mejorar la calidad de vida de las personas $y$, por lo tanto, la educación en el corto plazo propicia la equidad social; de manera que la inversión en educación tecnológica tenga posibilidades reales de traducirse en resultados cualitativa y cuantitativamente importantes, impactando de una forma social, cultural y económicamente en la región donde se localizan las Universidades Tecnológicas, así como también, en sus egresados, en sus comunidades de origen y por supuesto a través del tiempo, los resultados se verán reflejados en procesos hacia el mejoramiento de la calidad de vida de la regiones indígenas que forman parte del entorno donde se encuentras estos centros de educación superior.

\section{REFERENCIAS}

ANUIES. La educación superior en el siglo XXI. Líneas estratégicas de desarrollo. México: ANUIES, 2000.

CGTU Y 38 Universidades Tecnológicas. Universidades tecnológicas. Mandos medios para la Industria. México: SEP-CGUT/Noriega, 2000.

COORDINACIÓN GENERAL DE UNIVERSIDADES TECNOLÓGICAS (CGUT). 15 Años Universidadestecnológicas. México: SECRETARIA DE EDUCACION PUBLICA, 2006.

COORDINACIÓN GENERAL DE UNIVERSIDADES TECNOLÓGICAS (CGUT). La evaluación externa en las universidades tecnológicas. Un modelo eficaz para la rendición de cuentas. México: CGUT-SEP/LIMUSA/ UTHH, 2004.

CRESPO, P. F. La reforma de las universidades tecnológicas. ¿Un ciclo cerrado? Observatorío Ciudadano de la Educación, México, 4-5, 2009.

LORENZA VILLA LEVER, P. F.-C. Las universidades tecnológicas en el espejo de los institutos universitarios de tecnología franceses. Revista Mexicana de Investigación Educativa, México, p.17-49, 2002.

RAMSEY, C. Aprendiendo a trabajar. Una evaiuación del CONALEP y del Sistema de Universidades Tecnológicas en México. México: CONALEP, 2000 . 
RAMSEY, Gregor; CARNOY, Martin; WOODBURNE, Greg. Aprendiendo a trabajar: Una revisión del Colegio Nacional de Educación Profesional Técnica y del Sistema de Universidades Tecnológicas de México. México: Secretaria de Educación Publica, 2000.

RUIZ-LARRAGUIVEL, E. La educación superior tecnológica en México. Historia, situación actual y perspectivas. Revista Iberoamericana de Educación Superior (RIES) IISUE-UNAM/Universia, v. 2, n. 3, 2011. SECRETARÍA DE EDUCACIÓN PÚBLICA (SEP). Anuario estadístico. México: ANUIES, 2010.

UNESCO. Clasificación Internacional Normalizada de la Educación CINE. París: UNESCO, 2006. 